\title{
Entanglement entropy as a probe of the proximity effect in holographic superconductors
}

\author{
Xiao-Mei Kuang, ${ }^{a}$ Eleftherios Papantonopoulos ${ }^{a, b}$ and Bin Wang ${ }^{a}$ \\ ${ }^{a}$ INPAC, Department of Physics and Shanghai Key Lab for Particle Physics and Cosmology, \\ Shanghai Jiao Tong University, \\ Shanghai 200240, China \\ ${ }^{b}$ Department of Physics, National Technical University of Athens, \\ GR-15780 Athens, Greece \\ E-mail: kuangxiaomei@sjtu.edu.cn, lpapa@central.ntua.gr, \\ wang_b@sjtu.edu.cn
}

ABSTRACT: We study the entanglement entropy as a probe of the proximity effect of a superconducting system by using the gauge/gravity duality in a fully back-reacted gravity system. While the entanglement entropy in the superconducting phase is less than the entanglement entropy in the normal phase, we find that near the contact interface of the superconducting to normal phase the entanglement entropy has a different behavior due to the leakage of Cooper pairs to the normal phase. We verify this behavior by calculating the conductivity near the boundary interface.

KeYWORDS: Gauge-gravity correspondence, Holography and condensed matter physics (AdS/CMT)

ARXIV EPRINT: 1401.5720 


\section{Contents}

$\begin{array}{llr}1 & \text { Introduction } & 1\end{array}$

2 The gravitational sector $\quad 4$

3 The solutions of the holographic system 5

$\begin{array}{lll}4 & \text { The entanglement entropy of the holographic system } & 7\end{array}$

5 Conductivity 10

6 Conclusions 12

\section{Introduction}

The gauge/gravity duality has been proven to be a very powerful tool in studying strongly coupled phenomena using dual gravitational systems where the coupling is weak [1]. This duality which is well founded in string theory has many interesting applications and among them one which from the first sight it seems unexpected, in condensed matter physics (for a review, see [2]). A condensed matter system that is well studied using the gauge/gravity duality is the holographic superconductor.

The simplest holographic superconductor model which is extensively studied is described by an Einstein-Maxwell-scalar field theory with a negative cosmological constant [3, 4]. Its gravity sector is described by an Abelian-Higgs model with a stationary black hole metric which below a certain critical temperature the black hole acquires scalar hair. Its dual boundary field theory is described by a theory which is similar to the standard LandauGinzburg theory in which the scalar field corresponds to an operator, the order parameter, which condenses below the critical temperature, signalizing the onset of superconductivity.

This phenomenological approach has the virtue of simplicity, but does not capture all the underlying features of the gauge/gravity duality including quantum effects. This is hard to implement, as it remains a challenge to embed this model in a quantum system (string/M-theory). In spite of that, this holographic principle has been applied to many other condensed matter systems like the conventional and unconventional superfluids and superconductors [5], Fermi liquid behavior [6], non-linear hydrodynamics [7], quantum phase transitions [8] and transport [9]. In all these studies it is crucial to understand the low temperature limit. In the gravity sector this requires to go beyond the probe limit and to consider fully back-reacted gravitational systems. In the boundary theory the $T \rightarrow 0$ limit leads to strongly coupled systems in the quantum physics regime where new superfluid phenomena arise like for example the generation of inhomogeneous FFLO phases [11, 12]. 
Recently it was proposed [13] that this low temperature regime can be probed by the entanglement entropy $[14,15]$.

The entanglement entropy can be considered as a measure of how a given quantum system is strongly correlated (entangled). It was introduced as a tool to describe different phases and their corresponding phase transitions of a quantum system as the temperature goes to zero. The entanglement entropy is directly related to the degrees of freedom of a system, keeping track of them. It can also play the role of an order parameter of a phase transition at very low temperatures. The most important property of the entanglement entropy is that it is non-vanishing at zero temperature. For these reasons it was employed as a probe of quantum properties of the ground state for a given quantum system.

Recently the entanglement entropy was used to study various properties of holographic superconductors at low temperatures. In a model coming from $\mathcal{N}=8$ gauged supergravity [13] the entanglement entropy across the superconducting phase transition was studied. It was found that the entanglement entropy is lower in the superconducting phase than in the normal phase. This behavior was attributed to some kind of reorganization of the degrees of freedom of the system: because electrons are bounded in the superconducting phase to form Cooper pairs less degrees of freedom remain comparable to the normal phase.

The behavior of entanglement entropy across the holographic p-wave superconductor phase transition in an Einstein-Yang-Mills theory with a negative cosmological constant was studied in $[16,17]$. In [18] a holographic p-wave superconductor/insulator model was considered and it was found that as the back reaction increases, the transition is changed from second order to first order. Also in [19] the gravitational backreaction of the non-Abelian gauge field on the gravity dual to a $2+1 p$-wave superconductor was studied. It was found that the $p$-wave superconductor has lower entanglement entropy. The behavior of holographic entanglement entropy for imbalanced holographic superconductor was considered in [20]. It was found that entanglement entropy for this imbalanced system decreases with the increase of imbalance in chemical potentials.

In this work we will use the entanglement entropy as a probe of the proximity effect in a holographic superconductor. In condensed matter physics the proximity effect describes the dynamics of a system near the superconductor-normal metal interface where the superconducting electrons (Cooper pairs) may penetrate from the superconducting to normal phase. The leakage of the Cooper pairs weakens the superconductivity near the interface with a normal metal. This phenomenon can appear even in the absence of a magnetic field [21]. One of our motivations in this work is to construct a computationally tractable gravity model and show that it can reproduce basic properties of proximity effect in superconductivity.

The phenomenological analysis of the proximity effect was given by the generalized Ginzburg-Landau theory [21]. In this theory a complex scalar field $\Psi$ is considered as the superconducting order parameter which contributes to the free energy in the functional

$$
F_{G}=a(T)|\Psi|^{2}+\frac{b(T)}{2}|\Psi|^{4}+\gamma(T)|\vec{\nabla} \Psi|^{2}
$$

where the coefficient $a$ vanishes at the transition temperature $T_{c}$. At $T<T_{c}$, the coefficient $a$ is negative and the minimum of $F_{G}$ occurs for a uniform superconducting state 
with $|\Psi|^{2}=-a / b$. The coefficient $a$ is given by $a=\alpha\left(T-T_{c}\right)$, where $T_{c}$ is the critical temperature of the transition into the uniform superconducting state. The appearance of the proximity effect can simply be interpreted as the effect of the gradient term in the Ginzburg-Landau functional.

Let us consider the decay of the order parameter in the normal phase, i.e., at $T>$ $T_{c}$ assuming that our system is in contact with another superconductor with a higher critical temperature, and the $x$ axis is chosen perpendicular to the interface between the superconductor and the normal phases. The induced superconductivity is weak and, we use the linearized Ginzburg-Landau equation for the order parameter, $a \Psi-\gamma \frac{\partial^{2} \Psi}{\partial x^{2}}=0$, with $\gamma \neq 0$. The decaying solution is $\Psi=\Psi_{0} \exp [-x / \xi(T)]$, where $\xi(T)=\sqrt{\gamma / a}$ is the correlation length. When $\gamma>0$, the order parameter decays exponentially as $\Psi(\gamma>0)=$ $\Psi_{0} \exp [-x / \xi(T)]$. But when $\gamma<0$, close to the interface, the order parameter $\Psi(\gamma<0) \sim$ $\Psi_{0} \cos (x / \sqrt{|\gamma| / a})$, which is bigger than $\Psi(\gamma>0)$ in the normal phase close to the interface of the superconductor. This shows that due to the gradient term, the superconducting properties can be induced in the normal phase. This phenomenon is called the proximity effect. Simultaneously the leakage of the Cooper pairs weakens the superconductivity in the superconductor phase near the interface with a normal metal, which results in a decrease of the superconducting transition temperature in a thin superconducting layer in contact with a normal metal. Using the generalized Ginzburg-Landau functional [21], we see that the gradient term plays an important role in inducing the proximity effect.

Our aim here is to build a holographic superconductor with a dual boundary field described by a theory similar to the generalized Ginzburg-Landau theory. Such a theory, in the probe limit, was discussed in [22] in which a U(1) gauge field was introduced along with a complex scalar field coupled to a charged AdS black hole and a higher-derivative coupling between the $\mathrm{U}(1)$ gauge field and the scalar with coupling constant $\eta$. This coupling is provided by a potential term which in a covariant form reads $V(\Psi)=m^{2}|\Psi|^{2}+\eta\left|F^{\mu \nu} D_{\nu} \Psi\right|^{2}$, where $F=d A$ is the strength of a $\mathrm{U}(1)$ gauge field $A_{\mu}, \Psi$ is a charged complex scalar field of charge $q$ and mass $m$ and $D_{\mu}=\nabla_{\mu}-i q A_{\mu}$. However, this covariance is broken on the boundary, because the coefficient $\eta$ plays the role of $\gamma$ of the boundary Ginzburg-Landau theory and both of them indicate the strength of the gradient derivatives of the scalar field. What we had found in [22] is that, in the probe limit, large positive values of the coupling $\eta$ make easier the transition to a superconducting phase, while when $\eta$ becomes negative, it is more difficult for the condensation to be formed and this happens because the energy gap in the probe limit is larger for $\eta<0$ than the energy gap in the conventional case $(\eta=0)$.

One can expect that such high-derivatives terms can arise in string theory. Indeed, there are models based on exact solutions of $D=11$ and type IIB supergravity in which after consistent Kaluza-Klein truncations to four spacetime dimensions, fully back-reacted solutions describing holographic superconductors in three spacetime dimensions have been found $[23,24]$. These models, contain a large number of scalar, gauge fields and high derivatives of them, which need to be constrained in order to make the models tractable [25-27].

It is of great interest to generalize our previous study to the fully back-reacted theory. More interestingly, we would like to construct a tractable gravity model to reproduce the proximity effect in the holographic superconductor. Our $\eta$, which is analogous to $\gamma$ in the 
general Ginzburg-Landau functional [21], gives us the hope to build a holographic description of the proximity effect in the gravity model. Using the fact that the entanglement entropy plays the role of an order parameter, we will calculate it near the interface of superconducting/normal phase of a holographic superconductor in low temperatures and we will show that it gives us important information on the behavior of system. To verify this behavior we will also calculate the conductivity near the boundary interface.

The work is organized as follows. In section 2 we present the gravity sector of the model. In section 3 we present the fully back-reacted solution of the holographic system. In section 4 we discuss the entanglement entropy. In section 5 we calculate the conductivity and finally in section 6 are our conclusions.

\section{The gravitational sector}

We will consider a scalar field coupled to a U(1) gauge field with an action discussed in [22]

$$
S=\int d^{4} x \sqrt{-g}\left[\frac{R+6 / L^{2}}{16 \pi G}-\frac{1}{4} F_{\mu \nu} F^{\mu \nu}-\left|D_{\mu} \Psi\right|^{2}-V(\Psi)\right],
$$

where the potential term is given by the expression above. The field equations are given by:

- the Einstein equations

$$
R_{\mu \nu}-\frac{1}{2} R g_{\mu \nu}-\frac{3}{L^{2}} g_{\mu \nu}=8 \pi G T_{\mu \nu},
$$

- the Maxwell equations

$$
\begin{aligned}
& \nabla_{\mu} F^{\mu \nu}+\frac{\eta}{\sqrt{-g}} \partial_{\mu}\left[\sqrt{-g}\left(D_{\kappa} \Psi\right)\left(D_{\lambda} \Psi\right)^{*}\left(g^{\kappa \nu} F^{\mu \lambda}-g^{\kappa \mu} F^{\nu \lambda}+g^{\nu \lambda} F^{\mu \kappa}-g^{\mu \lambda} F^{\nu \kappa}\right)\right] \\
& =i q\left[\Psi^{*}\left(D^{\nu} \Psi\right)-\Psi\left(D^{\nu} \Psi\right)^{*}\right]+i q \eta g_{\mu \rho} F^{\rho \nu}\left[F^{\mu \kappa} \Psi^{*}\left(D_{\kappa} \Psi\right)-F^{\mu \lambda} \Psi\left(D_{\lambda} \Psi\right)^{*}\right],
\end{aligned}
$$

- and the scalar field equation

$$
\begin{aligned}
& -\frac{1}{\sqrt{-g}} \partial_{\mu}\left[\sqrt{-g} g^{\mu \nu}\left(\partial_{\nu} \Psi-i q A_{\nu} \Psi\right)\right]+i q g^{\mu \nu} A_{\nu}\left(\partial_{\mu} \Psi-i q A_{\mu} \Psi\right)+m^{2} \Psi \\
& =\frac{\eta}{\sqrt{-g}} \partial_{\mu}\left[\sqrt{-g} g_{\kappa \lambda} F^{\kappa \nu} F^{\lambda \mu}\left(\partial_{\nu} \Psi-i q A_{\nu} \Psi\right)\right]-i q \eta g_{\kappa \lambda} F^{\kappa \nu} F^{\lambda \mu} A_{\mu}\left(\partial_{\nu} \Psi-i q A_{\nu} \Psi\right)
\end{aligned}
$$

In this work, we will set $L=1,8 \pi G=1, q=1$.

We note that the presence of the coupling constant $\eta$ adds new terms in the field equations which makes the system of the differential equations highly non-trivial. We have to find numerical solutions of the fully back-reacted system. 


\section{The solutions of the holographic system}

We will generalize the probe limit discussion in [22] to a full back-reacted formalism by taking the ansatz of metric and matter fields as

$$
d s^{2}=-\frac{1}{z^{2}} f(z) e^{-\chi(z)} d t^{2}+\frac{1}{z^{2}}\left(d x^{2}+d y^{2}\right)+\frac{1}{z^{2}} \frac{d z^{2}}{f(z)}, \quad A_{\mu}=A_{t}(z) d t, \quad \Psi=\psi(z) .
$$

The temperature can be expressed as

$$
T=\left.\frac{-e^{\chi / 2} \partial_{z} f}{4 \pi}\right|_{z=z_{h}}
$$

Then, the independent Einstein-Maxwell equations reduced from (2.2) and (2.3) become

$$
\begin{aligned}
0= & \chi^{\prime}-\frac{z\left(1+2 e^{\chi} z^{4} \eta \mathrm{At}^{\prime 2}\right)\left(e^{\chi} q^{2} \mathrm{At}^{2} \psi^{2}+f^{2} \psi^{\prime 2}\right)}{f^{2}} \\
0= & f^{\prime}+\frac{3(1-f)}{z}-\frac{m^{2} \psi^{2}}{2 z}-\frac{e^{\chi} z^{3} A_{t}^{\prime 2}}{4}-\frac{e^{\chi} z q^{2} A_{t}^{2}\left(2+5 e^{\chi} z^{4} \eta A_{t}^{\prime 2}\right) \psi^{2}}{4 f}-\frac{z f\left(2+3 e^{\chi} \eta z^{4} A_{t}^{\prime 2}\right)}{4} \psi^{\prime 2} \\
0= & A_{t}^{\prime \prime}+\left[\frac{z^{2} f^{2} \chi^{\prime}+4 e^{\chi} \eta z^{3} \psi q^{2} A_{t}^{2}\left(z \psi f^{\prime}-2 f\left(\psi+z \psi^{\prime}+\frac{3}{4} z \psi \chi^{\prime}\right)\right)}{2 a_{0} f}\right. \\
& \left.+\frac{2 \eta z^{3} f \psi^{\prime}\left(z f^{\prime} \psi^{\prime}+2 f\left(\psi^{\prime}\left(1+\frac{1}{4} z \chi^{\prime}\right)+z \psi^{\prime \prime}\right)\right)}{a_{0}}\right] A_{t}^{\prime}-\left[\frac{2 \psi^{2} q^{2}\left(1+e^{\chi} \eta z^{4} A_{t}^{\prime 2}\right)}{a_{0}}\right] A_{t}
\end{aligned}
$$

while the scalar field equation (2.4) takes the form

$$
0=\psi^{\prime \prime}+\left[\frac{f^{\prime}}{f}+\frac{\left(4+z \chi^{\prime}\right)\left(1+e^{\chi} z^{4} \eta A_{t}^{\prime 2}\right)+4 z^{5} e^{\chi} \eta A_{t}^{\prime} A_{t}^{\prime \prime}}{2 z\left(e^{\chi} z^{4} \eta A_{t}^{\prime 2}-1\right)}\right] \psi^{\prime}+\left[\frac{e^{\chi} q^{2} A_{t}^{2}}{f^{2}}+\frac{m^{2}}{z^{2} f\left(e^{\chi} z^{4} \eta A_{t}^{\prime 2}-1\right)}\right] \psi,
$$

with $a_{0}=z^{2} f\left(1+2 \eta z^{2} f \psi^{\prime 2}\right)-2 e^{\chi} \eta z^{4} \psi^{2} q^{2} A_{t}^{2}$. Here (3.3) and (3.4) are the combination of $t t$ and $z z$ component of Einstein's equation and the $\mathrm{xx}$ component can be obtained from differentiating the two Einstein equations above.

Near the boundary $z \rightarrow 0$, we need $\chi(z=0)=0$ to recover the pure AdS boundary. The matter fields should behave as

$$
\begin{aligned}
\psi & =\psi^{(1)} z^{\Delta_{-}}+\psi^{(2)} z^{\Delta_{+}}+\cdots \\
A_{t} & =\mu-\rho z+\cdots
\end{aligned}
$$

where according to the AdS/CFT dictionary, $\psi^{(i)}=\left\langle O_{i}\right\rangle / \sqrt{2}, i=1,2$ and $O_{i}$ with the conformal dimensions $\Delta_{ \pm}=\frac{3}{2} \pm \frac{1}{2} \sqrt{9+4 m^{2}}$ are the corresponding dual operators of $\psi^{(i)}$ in the field theory side. $\mu$ and $\rho$ are the corresponding chemical potential and charge density in the dual boundary field theory, respectively. In this paper, we focus on the case $m^{2}=-2$ and set $\psi^{(2)}=0$ to consider $\psi^{(1)}$ as the vacuum expectation value of the operator $\left\langle O_{1}\right\rangle$. 


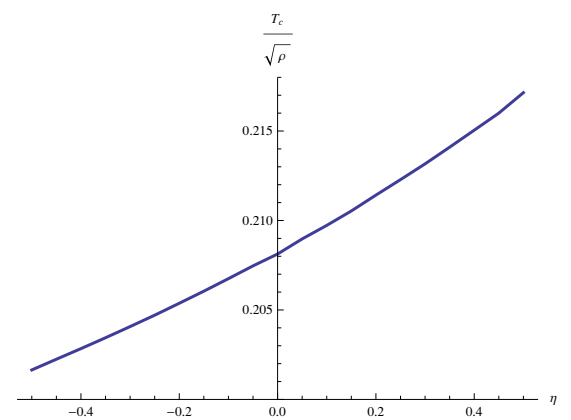

Figure 1. The dependence of critical temperature on the coupling constant $\eta$.

At the horizon $z=z_{h}$, the regular condition implies $A_{t}\left(z_{h}\right)=0$ and $f\left(z_{h}\right)=0$. Then we can expand all the fields near the horizon and use the scaling symmetries

$$
\begin{aligned}
& t \rightarrow a t, \quad z \rightarrow a z, \\
& z \rightarrow a z, \quad(t, x, y) \rightarrow(t, x, y) / a, \quad A_{t} \rightarrow a A_{t}, \quad f \rightarrow f, \quad \psi \rightarrow \psi,
\end{aligned}
$$

to set $z_{h}=1$.

At high temperature, the scalar field will vanish and there is no condensation. The solution is the Reissner-Nordström AdS black hole with

$$
\chi=\psi=0, \quad A_{t}=\mu\left(1-\frac{z}{z_{h}}\right), \quad f=1-\frac{z^{3}}{z_{h}^{2}}\left(1+\frac{\mu^{2} z_{h}^{2}}{4}\right)+\frac{z^{4}}{z_{h}^{4}}\left(\frac{\mu^{2} z_{h}^{2}}{4}\right) .
$$

The temperature is give by

$$
T=\frac{1}{4 \pi z_{h}}\left(3-\frac{\mu^{2} z_{h}^{2}}{4}\right) .
$$

When the temperature decreases to be lower than a critical value, a new type of charged black hole with non-vanishing charged scalar profile is numerically available. This corresponds to a hairy phase with $O_{1}$ non-vanishing. The explicit dependence of the critical temperature for $O_{1}$ on the coupling is presented in figure 1 . We see that the critical temperature increases as the coupling $\eta$ becomes larger, which is consistent with the results in the probe limit in our previous work [22]. Moreover, we show the vacuum expectation values for $O_{1}$ in figure 2. It is observed that as the coupling increases, the condensation gap is lower which agrees well with the property of the critical temperature we discussed above. Thus, from the gravitational side, we have found that the greater strength of the interaction will make the condensation easier to form in the back-reacted background. In the dual boundary field theory, this means that with stronger coupling between the $U(1)$ gauge field and the scalar field, the gauge symmetry can be broken more easily.

Having the solutions of the normal phase and the hairy phase below the critical temperature, we will study the holographic entanglement entropy (HEE) from high to lower temperatures in the next section. 


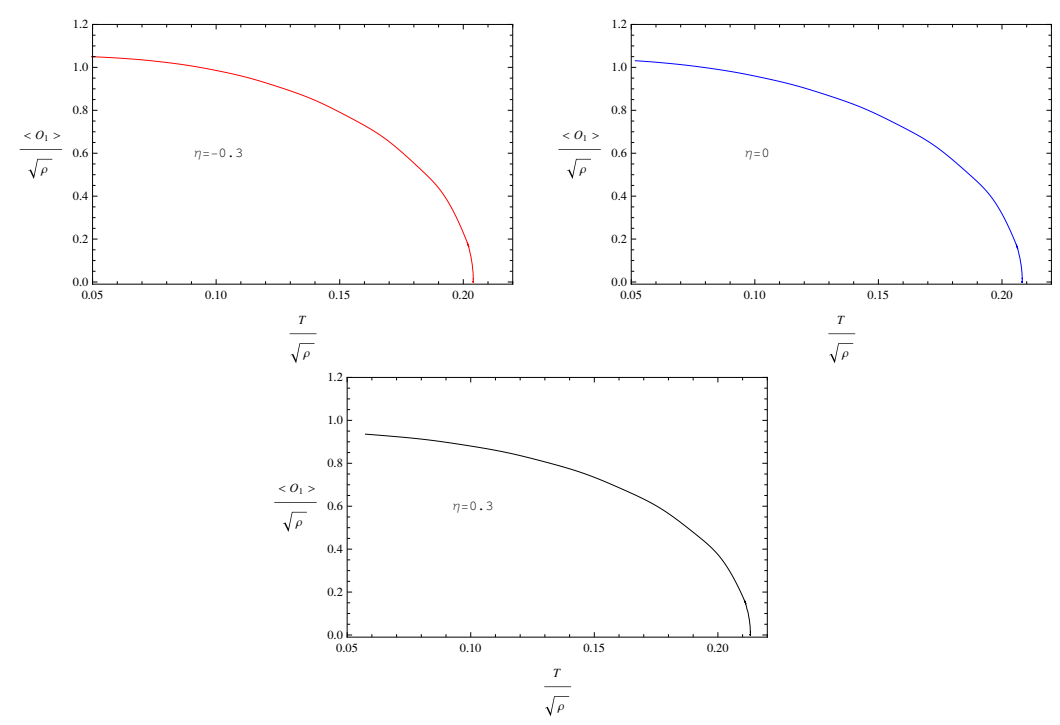

Figure 2. Plots of the operator $O_{1}$ versus temperature after condensation with different values of the coupling constant $\eta$.

\section{The entanglement entropy of the holographic system}

Let us now discuss how we can incorporate the notion of entanglement entropy in the AdS/CFT correspondence. Imagine that we have a system $A$ in the boundary CFT which has a gravity dual. Since the information included in a subsystem $B$ is evaluated by the entanglement entropy $S_{A}$, we can ask which part of AdS space is responsible for the calculation of $S_{A}$ in the dual gravity side. In [14] a formula was proposed

$$
S_{A}=\frac{\operatorname{Area}\left(\gamma_{A}\right)}{4 G_{N}^{(d+2)}},
$$

where $\gamma_{A}$ is the $d$-dimensional minimal surface whose boundary is given by the $(d-1)$ dimensional manifold $\partial \gamma_{A}=\partial A$. The constant $G_{N}^{(d+2)}$ is the Newton constant of the general gravity in $\mathrm{AdS}_{d+2}$.

Before studying the holographic entanglement entropy (HEE) in this holographic model, we will geometrize the HEE of (4.1) in terms of AdS/CFT duality. Following [13], we will consider the subsystem $A$ with a straight strip geometry described by $-\frac{l}{2} \leq x \leq$ $\frac{l}{2}, 0 \leq y \leq L$, where $l$ is defined as the size of region $A$ and $L$ is a regulator which can be set to be infinity. The induced metric of the hypersurface $\gamma_{A}$ whose boundary is the same as the stripe and has a profile like (3.1) reads as

$$
d s_{\text {induced }}^{2}=\frac{1}{z^{2}}\left[\left(\frac{1}{f}+x^{\prime}(z)\right) d z^{2}+d y^{2}\right] .
$$

Thus, the HEE connecting with the area of the surface can be expressed as

$$
4 G_{4} S=\operatorname{Area}\left(\gamma_{A}\right)=L \int_{-l / 2}^{l / 2} \frac{d x}{z^{2}} \sqrt{1+\frac{z^{\prime}(x)^{2}}{f}} .
$$



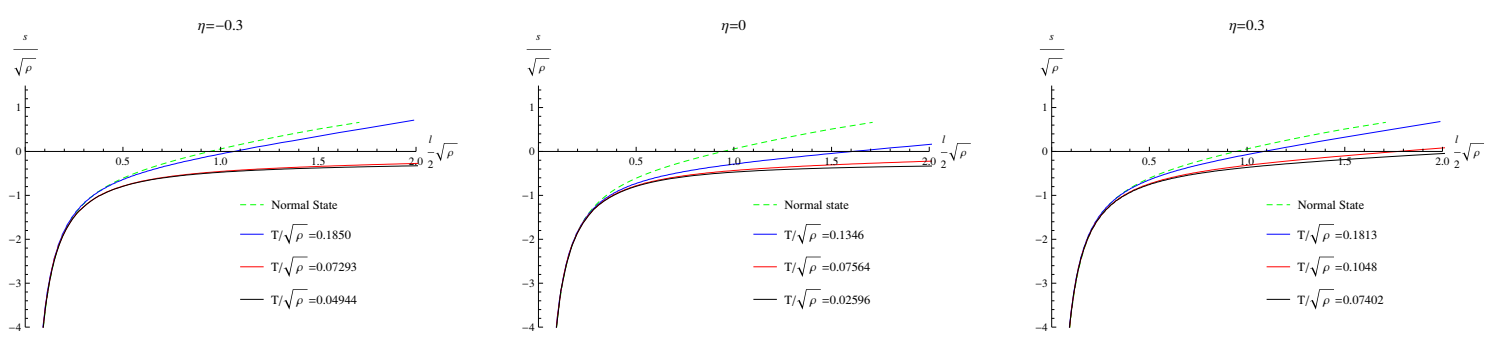

Figure 3. The entanglement entropy as a function of stripe width for various temperatures and different values of the coupling $\eta$.

The above expression can be treated as the Lagrangian with $x$ direction thought of as time. The corresponding Hamiltonian is conserved because the Lagrangian does not explicitly depend on $x$. So we can get a constant of motion as

$$
\frac{1}{z_{*}^{2}}=\frac{1}{z^{2} \sqrt{1+\frac{z^{\prime}(x)^{2}}{f}}},
$$

with $z_{*}$ satisfying the condition $d z /\left.d x\right|_{z=z_{*}}=0$. From (4.4), we can write the width $l$ in terms of $z_{*}$

$$
\frac{l}{2}=\int_{\epsilon}^{z_{*}} d z \frac{z^{2}}{\sqrt{\left(z_{*}^{4}-z^{4}\right) f}} .
$$

Substituting (4.4) into (4.3), we can obtain the entanglement entropy

$$
4 G_{4} S=2 L \int_{\epsilon}^{z_{*}} d z \frac{z_{*}^{2}}{z^{2}} \frac{1}{\sqrt{\left(z_{*}^{4}-z^{4}\right) f}}=2 L\left(s+\frac{1}{\epsilon}\right) .
$$

Here the term $1 / \epsilon$ is divergent. While the term $s$ is a finite term, so it is physically important.

Now, we can calculate the entanglement entropy $s$ based on the solution discussed in the last section. We will see the behavior of HEE from normal phase to hairy phase and investigate the effect of the $\eta$ coupling. Note that according to the scaling symmetries in (3.9), the dimensionless quantities are $T / \sqrt{\rho}, \sqrt{\rho} l$ and $s / \sqrt{\rho}$.

Fixing the temperature, we see in figure 3 how the HEE changes as the width of stripe $l$ changes. The green line is for the normal phase with the RN-AdS black hole background. We see that below the critical temperature when the scalar field starts to condensate, the entanglement entropy becomes smaller and it drops when the temperature becomes lower. This is consistent with the expectation that in the superconducting phase the degrees of freedom decrease due to the formation of Cooper pairs [13]. This property holds for different values of the coupling $\eta$.

To illustrate the influence of the $\eta$ coupling, we present the HEE in change of temperature for a fixed $l$ in figure 4. The light green line is the HEE for the normal state with RN-AdS black hole background. As the temperature decreases, the slope of HEE presents a discontinuous change at a critical temperatures $T_{c}$ denoted by vertical dashed 


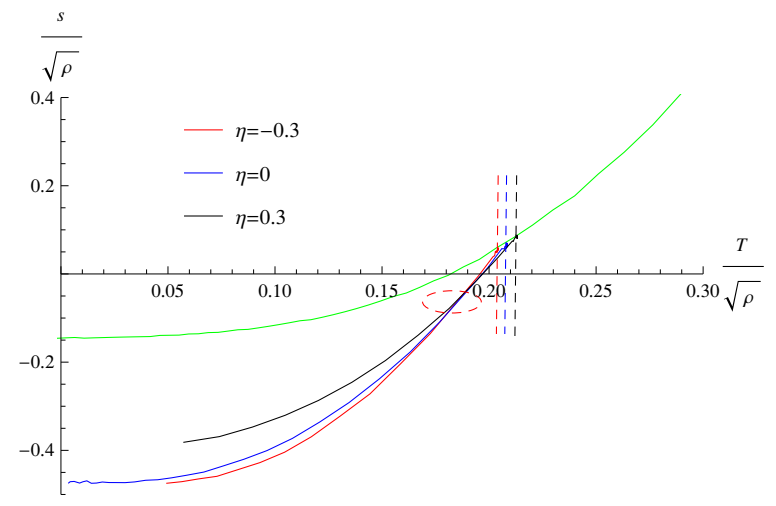

Figure 4. The entanglement entropy as a function of temperature for a fixed $\sqrt{\rho} \frac{l}{2}=1$. The green curve depicts the normal phase while the other curves depict the superconducting phases with different values of the coupling $\eta$. The region in the ellipse is enlarged in figure 5 .

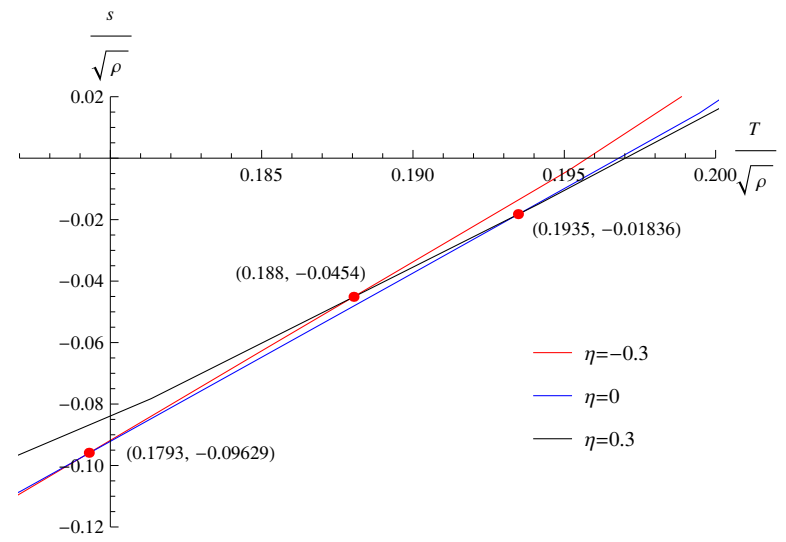

Figure 5. The region in the ellipse in figure 4 is shown here enlarged. As exhibited in the plot, the curves of HEE for the coupling $\eta=0$ intersects with that for $\eta=-0.3$ and $\eta=0.3$ at $T / \sqrt{\rho}=0.1793$ and $T / \sqrt{\rho}=0.1935$, respectively. While the curves with the couplings $\eta=-0.3$ and $\eta=0.3$ intersect at $T / \sqrt{\rho}=0.188$. This sharp change of HEE near the interface is due to the proximity effect.

lines in the figure for different strength of the coupling $\eta$. The discontinous change of the HEE marks the phase transition point from the normal state to the superconducting state. We again observe that the superconducting phase always have smaller HEE after the phase transition.

At low temperature, the HEE of a superconductor with a smaller $\eta$ is smaller. Physically this happens because at low temperature the condensation becomes stronger with higher condensation gap for smaller coupling $\eta$ so the number of Cooper pairs is increased, which results in less degrees of freedom available.

However this property does not hold when the temperature increases near the critical value, which is close to the interface with the normal phase, marked in the ellipse in figure 4, which is enlarged in figure 5 . When the temperature is increased above $T / \sqrt{\rho}=0.1793$, we see that the HEE for $\eta=-0.3$ becomes higher than the case with $\eta=0$. When the 
temperature is above $T / \sqrt{\rho}=0.188$, the HEE for $\eta=-0.3$ surpasses the value for $\eta=0.3$. The sharp change of the HEE which is related to the coupling $\eta$ in the vicinity of the contact interface, is not trivial. This behavior can be attributed to the proximity effect.

For the smaller coupling holographic superconductor, it is more likely that the Cooper pairs of the supercontacting state can penetrate the normal state, which effectively results in the increase of the HEE in the superconductor phase. This is a similar behavior to the generalized Ginzburg-Landau theory on the boundary, where the phenomenological coefficient $\gamma$ is related to the correlation length $\xi=\sqrt{\gamma /|a|}$ as we discussed in the introduction. A more negative $\gamma$ for a fixed $a$ leads more Cooper pairs to penetrate and this results in a larger leftover order parameter in the normal phase [21]. On the other hand, for larger positive values of the coupling $\eta$ coupling, Cooper pairs remain in the superconductor phase, thus leads the HEE to relatively smaller values. Again this agrees to the generalized Ginzburg-Landau theory description that more positive $\gamma$ leads to smaller penetration of the Cooper pairs so that the leftover order parameter in the normal phase is weaker.

\section{Conductivity}

In this section we will discuss the conductivity in an attempt to understand better the behavior of the system near the critical temperature. In the superconductor, the conductivity possesses certain distinguishing properties which are largely dependent on the microscopic details. To support the results we found studying the entanglement entropy, we will calculate the real part of conductivity for two characteristic temperatures at which we observed two different behaviors of the entanglement entropy. The first one is a low temperature which characterizes the superconducting phase where the entanglement entropy decreases with the decrease of the coupling constant $\eta$, at which more Cooper pairs are formed for more negative coupling $\eta$. For a high temperature we choose a temperature at which the superconducting state had just been formed and effectively it is close to the transition temperature of the superconducting to normal state. At the high temperature we found that the entanglement entropy is higher for negative $\eta$.

We consider the perturbation of metric and $\mathrm{U}(1)$ field as $\delta g_{\mu \nu}=g_{t x}(z) e^{-i \omega t}$ and $\delta A_{\mu}=A_{x}(z) e^{-i \omega t}$. Then, the first order perturbation equation of $g_{t x}$ can be deduced as

$$
g_{t x}^{\prime}-\frac{2 g_{t x}}{z}-A_{x} A_{t}^{\prime}\left[1-z^{2} \eta\left(f \psi^{\prime 2}-\frac{q^{2} e^{\chi} A_{t}^{2} \psi^{2}}{f}\right)\right]=0
$$

Having this equation we can deduce the linearised perturbative Maxwell equation which decouples from $g_{t x}$

$$
\left(1+2 z^{2} \eta f \psi^{\prime 2}\right) A_{x}^{\prime \prime}+\left(\frac{f^{\prime}}{f}-\frac{\chi^{\prime}}{2}+a_{1} \eta\right) A_{x}^{\prime}+\left[\left(\frac{\omega^{2}}{f^{2}}-\frac{z^{2} A_{t}^{\prime 2}}{f}\right) e^{\chi}-\frac{2 q^{2} \psi^{2}}{z^{2} f}+a_{2} \eta+a_{3} \eta^{2}\right] A_{x}=0
$$


with

$$
\begin{aligned}
a_{1}= & z \psi^{\prime}\left[z \psi^{\prime}\left(4 f^{\prime}-f \chi^{\prime}\right)+4 f\left(\psi^{\prime}+z \psi^{\prime \prime}\right)\right], \\
a_{2}= & \frac{e^{2 \chi} q^{2} z^{2} A_{t}^{2} \psi^{2}}{f^{2}}\left(z^{2} A_{t}^{\prime 2}-\frac{2 \omega^{2}}{f}\right) \\
& -\frac{e^{\chi}}{f}\left[2 q^{2} z^{2} A_{t} \psi A_{t}^{\prime \prime}+z A_{t}^{\prime 2}\left(2 q^{2} \psi^{2}+z^{2} \psi^{\prime} f\right)+q^{2} z A_{t} \psi A_{t}^{\prime}\left(4 \psi+4 z \psi^{\prime}+z \psi \chi^{\prime}\right)\right], \\
a_{3}= & 2 e^{\chi} z^{6} A_{t}^{\prime 2} f\left[\psi^{\prime 4}-2 e^{\chi}\left(\frac{q A_{t} \psi \psi^{\prime}}{f}\right)^{2}+e^{2 \chi}\left(\frac{q A_{t} \psi}{f}\right)^{4}\right] .
\end{aligned}
$$

Although that above equation seems very complicated, it can be solved by imposing the ingoing boundary condition near the horizon

$$
A_{x}\left(z \rightarrow z_{h}\right) \propto f^{\frac{-i \omega}{4 \pi T}}
$$

where the temperature $T$ is defined in (3.2). Near the asymptotic AdS boundary, the behavior of perturbation $A_{x}$ is

$$
A_{x}(z \rightarrow 0)=A_{x}^{(0)}+z A_{x}^{(1)} .
$$

Then, by invoking the AdS/CFT duality, the conductivity of holographic superconductivity can be expressed as [3]

$$
\sigma=-\frac{i A_{x}^{(1)}}{\omega A_{x}^{(0)}} .
$$

After solving the equation with the boundary condition (5.3), we can numerically extract the asymptotic value of $A_{x}$ to calculate the conductivity of our system.

We concentrate on the real part of the conductivity, since it is the dissipative part of the conductivity and measures the presence of charged states as a function of energy. At the low temperature, for example when $T / \sqrt{\rho}=0.0574$, which is lower than the smallest crossing temperature in figure 5 , we see in the left panel of figure 6 that at low frequencies the holographic superconducting system with smaller coupling $\eta$ has lower real part of conductivity and a larger frequency gap. The gap in the conductivity depends on the condensation, $\omega_{g} \sim\langle O\rangle$, which indicates a gap in the spectrum of charged excitations. For the smaller coupling, the higher condensation leads to the larger superconducting gap and to a smaller real part of the conductivity. The drop in the real part of the conductivity corresponds to a drop in the density of excitations at energies below the chemical potential. Thus for smaller $\eta$, more 'electrons' are bounded in Cooper pairs which explains the smaller HEE at low temperature.

At high temperature near the critical point, where the dependence of HEE on the coupling $\eta$ is reversed compared with the low temperature case, the behavior of the real part of the conductivity is plotted in the right plot of figure 6 with $T / \sqrt{\rho}=0.20407$ for example, which is higher than the largest crossing temperature in figure 5 . In the low frequency, we see that the real part of conductivity is bigger when the coupling is smaller. 

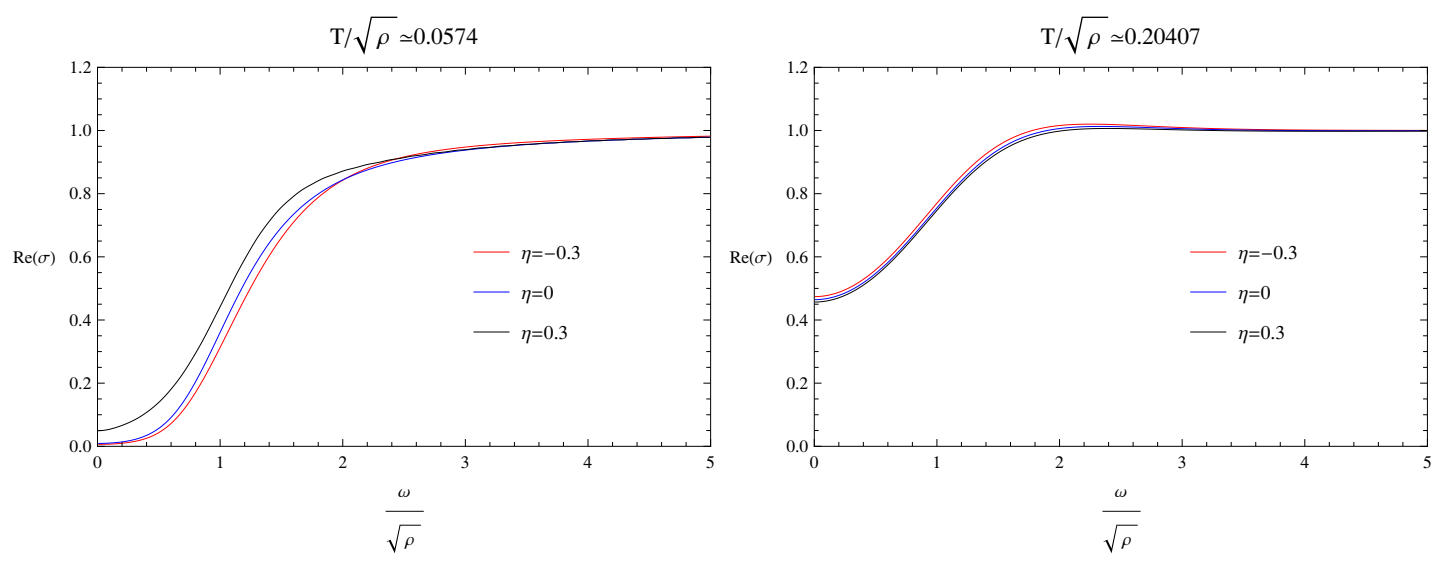

Figure 6. The real part of the conductivity with $T=0.0574 \sqrt{\rho}$ and $T=0.20407 \sqrt{\rho}$.

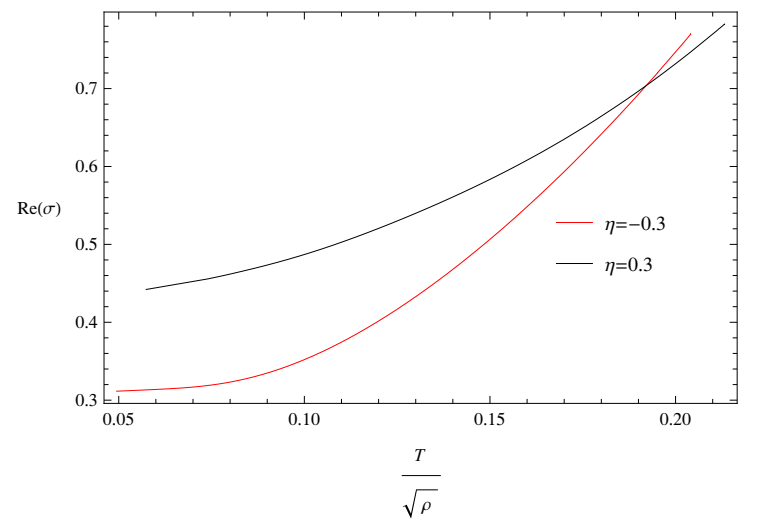

Figure 7. Dependence of the real part of the conductivity on the temperature.

Fixing the frequency, we show the dependence of the real part of the conductivity on the temperature in figure 7 . The sharp different dependence of the real part of the conductivity on the coupling $\eta$ at low and high temperatures are clearly shown. In the contact interface, we see that for the smaller $\eta$, the real part of the conductivity is higher. The bigger real part of the conductivity in the contact interface supports the proximity effect argument that less Cooper pairs are left in the superconducting phase with smaller $\eta$ coupling.

\section{Conclusions}

We have studied the behavior of the entanglement entropy of a superconducting system. Motivated by the generalized Ginzburg-Landau theory we have introduced a higherderivative coupling between the $\mathrm{U}(1)$ gauge field and the scalar field with coupling constant $\eta$. In the boundary theory this coupling corresponds to one of the phenomenological constants of the Landau-Ginzburg theory. We have solved numerically the fully back-reacted gravitational system and found that as the coupling $\eta$ increases the critical temperature increases and the energy gap decreases. This result suggests that the system with larger coupling is easier to enter the superconducting phase. 
Knowing the behavior of the system below the critical temperature, we have calculated the entanglement entropy using a stripe geometry. We have found that the entanglement entropy is less than the entanglement entropy of the normal phase. This agrees with the known results that the entanglement entropy plays the role of the order parameter of the superconducting system counting the degrees of freedom. In the superconducting phase less degrees of freedom are available because of the formation of Cooper pairs, therefore the entanglement entropy is less than the normal phase. We also found that a larger coupling gives a larger entanglement entropy. This means that for a fixed temperature for a larger coupling, less Cooper pairs are available in the superconducting system.

We have found that near the boundary superconducting/normal interface the entanglement entropy has reversed behavior. More negative coupling gives higher entanglement entropy. This can be explained because of the proximity effect. For the more negative coupling, more Cooper pairs have leaked to the normal phase, so that less electrons are bounded in the superconductor phase which leads to the higher value of the entanglement entropy. This behavior of the entanglement entropy near the boundary contact interface is very interesting. It further supports the previous finding that the entanglement entropy plays the role of the order parameter, measuring the degrees of freedom of a superconducting system.

To support further this behavior of the entanglement entropy we have calculated the real part of conductivity for two characteristic temperatures, one low temperature and the other near the critical temperature which effectively close to the interface of the normal phase. For the low frequency at the low temperature, smaller coupling has lower conductivity. But when the temperature is near the critical temperature, the behavior of the conductivity is reversed due to the proximity effect.

In conclusion we have built a holographic superconductor and presented a holographic description of the proximity effect in superconductivity. It would be interesting to extend this study in the presence of an external magnetic field. For an inhomogeneous magnetic field the entanglement entropy can give us more information on the phase structure of the holographic superconducting system at low temperatures and possibly of the formation of FFLO states.

\section{Acknowledgments}

This work is supported partially by the NNSF of China and the Shanghai Science and Technology Commission under Grant No. 11DZ2260700. E.P is supported partially by ARISTEIA II action of the operational programme education and long life learning which is co-funded by the European Union (European Social Fund) and National Resources.

Open Access. This article is distributed under the terms of the Creative Commons Attribution License (CC-BY 4.0), which permits any use, distribution and reproduction in any medium, provided the original author(s) and source are credited. 


\section{References}

[1] J.M. Maldacena, The Large-N limit of superconformal field theories and supergravity, Adv. Theor. Math. Phys. 2 (1998) 231 [Int. J. Theor. Phys. 38 (1999) 1113] [hep-th/9711200] [INSPIRE].

[2] S.A. Hartnoll, Lectures on holographic methods for condensed matter physics, Class. Quant. Grav. 26 (2009) 224002 [arXiv:0903.3246] [INSPIRE].

[3] S.A. Hartnoll, C.P. Herzog and G.T. Horowitz, Building a Holographic Superconductor, Phys. Rev. Lett. 101 (2008) 031601 [arXiv:0803.3295] [INSPIRE].

[4] S.A. Hartnoll, C.P. Herzog and G.T. Horowitz, Holographic Superconductors, JHEP 12 (2008) 015 [arXiv:0810.1563] [InSPIRE].

[5] S.S. Gubser and S.S. Pufu, The Gravity dual of a p-wave superconductor, JHEP 11 (2008) 033 [arXiv:0805.2960] [INSPIRE].

[6] M. Cubrovic, J. Zaanen and K. Schalm, String Theory, Quantum Phase Transitions and the Emergent Fermi-Liquid, Science 325 (2009) 439 [arXiv:0904.1993] [INSPIRE]

[7] S. Bhattacharyya, V.E. Hubeny, S. Minwalla and M. Rangamani, Nonlinear Fluid Dynamics from Gravity, JHEP 02 (2008) 045 [arXiv:0712.2456] [INSPIRE].

[8] N. Iqbal, H. Liu, M. Mezei and Q. Si, Quantum phase transitions in holographic models of magnetism and superconductors, Phys. Rev. D 82 (2010) 045002 [arXiv: 1003.0010] [INSPIRE].

[9] C.P. Herzog, P. Kovtun, S. Sachdev and D.T. Son, Quantum critical transport, duality and M-theory, Phys. Rev. D 75 (2007) 085020 [hep-th/0701036] [INSPIRE].

[10] G.T. Horowitz and M.M. Roberts, Zero Temperature Limit of Holographic Superconductors, JHEP 11 (2009) 015 [arXiv:0908.3677] [INSPIRE].

[11] J. Alsup, E. Papantonopoulos and G. Siopsis, FFLO States in Holographic Superconductors, arXiv: 1208.4582 [INSPIRE].

[12] J. Alsup, E. Papantonopoulos and G. Siopsis, A Novel Mechanism to Generate FFLO States in Holographic Superconductors, Phys. Lett. B 720 (2013) 379 [arXiv:1210.1541] [InSPIRE].

[13] T. Albash and C.V. Johnson, Holographic Studies of Entanglement Entropy in Superconductors, JHEP 05 (2012) 079 [arXiv: 1202.2605] [INSPIRE].

[14] S. Ryu and T. Takayanagi, Holographic derivation of entanglement entropy from AdS/CFT, Phys. Rev. Lett. 96 (2006) 181602 [hep-th/0603001] [INSPIRE].

[15] S. Ryu and T. Takayanagi, Aspects of Holographic Entanglement Entropy, JHEP 08 (2006) 045 [hep-th/0605073] [INSPIRE].

[16] R.-G. Cai, S. He, L. Li and Y.-L. Zhang, Holographic Entanglement Entropy on P-wave Superconductor Phase Transition, JHEP 07 (2012) 027 [arXiv: 1204.5962] [INSPIRE].

[17] R.-G. Cai, S. He, L. Li and Y.-L. Zhang, Holographic Entanglement Entropy in Insulator/Superconductor Transition, JHEP 07 (2012) 088 [arXiv:1203.6620] [INSPIRE].

[18] R.-G. Cai, L. Li, L.-F. Li and R.-K. Su, Entanglement Entropy in Holographic P-Wave Superconductor/Insulator Model, JHEP 06 (2013) 063 [arXiv:1303.4828] [INSPIRE].

[19] R.E. Arias and I.S. Landea, Backreacting p-wave Superconductors, JHEP 01 (2013) 157 [arXiv: 1210.6823] [INSPIRE]. 
[20] A. Dutta and S.K. Modak, Holographic entanglement entropy in imbalanced superconductors, JHEP 01 (2014) 136 [arXiv: 1305.6740] [INSPIRE].

[21] A.I. Buzdin, Proximity effects in superconductor-ferromagnet heterostructures, Rev. Mod. Phys. 77 (2005) 935,

[22] X.-M. Kuang, E. Papantonopoulos, G. Siopsis and B. Wang, Building a Holographic Superconductor with Higher-derivative Couplings, Phys. Rev. D 88 (2013) 086008 [arXiv: 1303.2575] [INSPIRE].

[23] J.P. Gauntlett, S. Kim, O. Varela and D. Waldram, Consistent supersymmetric Kaluza-Klein truncations with massive modes, JHEP 04 (2009) 102 [arXiv:0901.0676] [INSPIRE].

[24] J.P. Gauntlett, J. Sonner and T. Wiseman, Holographic superconductivity in M-theory, Phys. Rev. Lett. 103 (2009) 151601 [arXiv:0907.3796] [INSPIRE].

[25] J.P. Gauntlett, J. Sonner and T. Wiseman, Quantum Criticality and Holographic Superconductors in M-theory, JHEP 02 (2010) 060 [arXiv:0912.0512] [INSPIRE].

[26] N. Bobev, N. Halmagyi, K. Pilch and N.P. Warner, Supergravity Instabilities of Non-Supersymmetric Quantum Critical Points, Class. Quant. Grav. 27 (2010) 235013 [arXiv: 1006.2546] [INSPIRE].

[27] N. Bobev, A. Kundu, K. Pilch and N.P. Warner, Minimal Holographic Superconductors from Maximal Supergravity, JHEP 03 (2012) 064 [arXiv: 1110.3454] [INSPIRE]. 